\title{
Physical activity and nutrition behavioural outcomes of a home-based intervention program for seniors: a randomized controlled trial
}

\author{
Linda Burke ${ }^{1 *}$, Andy H Lee ${ }^{1}$, Jonine Jancey ${ }^{1}$, Liming Xiang ${ }^{2}$, Deborah A Kerr ${ }^{1}$, Peter A Howat ${ }^{1,3}$, \\ Andrew P Hills ${ }^{4}$ and Annie S Anderson ${ }^{5}$
}

\begin{abstract}
Background: This intervention aimed to ascertain whether a low-cost, accessible, physical activity and nutrition program could improve physical activity and nutrition behaviours of insufficiently active 60-70 year olds residing in Perth, Australia.

Methods: A 6-month home-based randomised controlled trial was conducted on 478 older adults (intervention, $\mathrm{n}=248$; control, $\mathrm{n}=230$ ) of low to medium socioeconomic status. Both intervention and control groups completed postal questionnaires at baseline and post-program, but only the intervention participants received project materials. A modified fat and fibre questionnaire measured nutritional behaviours, whereas physical activity was measured using the International Physical Activity Questionnaire. Generalised estimating equation models were used to assess the repeated outcomes over both time points.
\end{abstract}

Results: The final sample consisted of 176 intervention participants and 199 controls (response rate 78.5\%) with complete data. After controlling for demographic and other confounding factors, the intervention group demonstrated increased participation in strength exercise $(p<0.001)$, walking $(p=0.029)$ and vigorous activity $(p=$ $0.015)$, together with significant reduction in mean sitting time $(p<0.001)$ relative to controls. Improvements in nutritional behaviours for the intervention group were also evident in terms of fat avoidance $(p<0.001)$, fat intake $(p=0.021)$ and prevalence of frequent fruit intake $(p=0.008)$.

Conclusions: A minimal contact, low-cost and home-based physical activity program can positively influence seniors' physical activity and nutrition behaviours.

Trial registration: anzctr.org.au Identifier: ACTRN12609000735257

Keywords: Fat avoidance, Fibre intake, Fruit intake, Goal setting, Sitting, Strength exercise, Vegetable intake, Walking

\section{Background}

Physical activity is known to decline with age [1]. In Australia, $51 \%$ of the older population aged 60 to 75 years are insufficiently active, with the highest prevalence of inactive behaviour being reported in adults over 75 years of age [2]. Similarly, rates of physical activity among American adults aged 65 years and older are low, with only $20 \%$ of women and $25 \%$ of men meeting the national recommended physical activity guidelines [3],

\footnotetext{
* Correspondence: Iburke67@gmail.com

'School of Public Health, Curtin University, Perth, WA, Australia

Full list of author information is available at the end of the article
}

while $26 \%$ of those in the $65-74$ age group are inactive [4]. Research has demonstrated that sedentary behaviours may be linked to obesity, cardiovascular diseases and type 2 diabetes [5-11]. Moreover, as people age, their nutritional requirements change and energy requirements decrease. Older adults should consume nutritious foods that are high in fibre and low in saturated fats to help maintain a healthy weight [12]. However, worldwide trends are shifting towards an increased consumption of energy-dense foods rich in saturated fats and sugars [13], leading to energy imbalance and rise in diet related diseases [14].

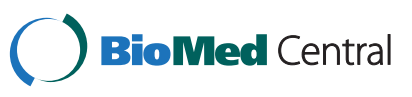


In the literature, intervention programs designed to improve physical activity levels or dietary habits have used a variety of strategies including workbooks, calendars, telephone counselling, goal setting and pedometers [15-19]. Although interventions combining physical activity and nutrition appear to result in better outcomes than those focusing on either aspect alone $[17,19]$, there is limited evidence on home-based interventions in terms of improving both physical activity and nutritional behaviours among people aged 60-70 years [20-22]. Moreover, research involving seniors has generally been undertaken with a small sample size $[18,19,23]$, or targeting those with a specific chronic disease $[15,19,23]$. Another limitation is that the participants recruited were generally self-referrals and volunteers $[17,18,24]$ as opposed to being randomly selected samples. Therefore, there is an urgent need to develop well-designed interventions that overcome such shortcomings [25].

The Physical Activity and Nutrition for Seniors (PANS) program attempted to improve both physical activity and nutritional behaviours. It was a low-cost and accessible home-based intervention targeting insufficiently active low to middle income older adults aged 60-70 years who could semi-tailor the program to suit their own pace and needs [26]. We targeted these "baby boomers" (60-70 year olds) because they contribute to the fast growing segment of the population who are retired or near retirement. The aim of the present study was to determine whether the PANS intervention was effective with respect to the main outcome measures of self-reported physical activity and nutritional behaviours. The findings have important implications for the control and prevention of overweight and obesity in the older population.

\section{Methods Study design}

PANS was a 6-month two-arm randomised controlled trial collecting data at two time points (baseline; post intervention). The project protocol was approved by the Human Research Ethics Committee of Curtin University (approval number HR 186/2008) and written consent was obtained from all participants.

\section{Procedure}

A random sample of 478 participants was recruited from 60 suburbs/neighbourhoods (30 intervention; 30 controls) within the metropolitan area of Perth, the capital of Western Australia. Participants were randomly selected from the Australian Federal Electoral Roll in 2010, which provided a representative sampling frame. Suburbs were required to comprise at least $14 \%$ of adults aged 60 years and older; contain at least 120 potential participants; and be classified as low or medium socioeconomic status [27]. Participant selection criteria called for "insufficiently active" recruits who participated in less than 30 minutes of moderate-intensity physical activity on at least 5 days per week [28]; aged 60 to 70 years; considered "healthy" to the extent that participation in a low-stress physical activity program would not place them at risk; and not on any special diet [26]. Participant flow and corresponding sample sizes are presented in Figure 1. Of the 248 program participants and 230 controls that completed the baseline questionnaire, 176 and 199 seniors respectively with complete data were available for analysis, giving a final response rate of $78.5 \%$.

\section{Intervention}

The intervention was developed using Social Cognitive Theory $[29,30]$ and the Precede-Proceed Model [31] incorporating voluntary cooperation and self-efficacy [29,32], and based on a pilot project [33] that produced encouraging results with respect to adherence and behaviour change [33,34]. Further revisions were made after extensive formative research [26]. Such formative data from representatives of the target group confirmed the preference for a flexible, home-based program, whereby participants would be able to set their own goals and semi-tailor the intervention to better suit their own needs [26].

The main resource of the home-based program was a booklet specially designed for seniors that provided physical activity and nutrition recommendations and encouraged goal setting. The booklet was supported by an exercise chart, calendar, bi-monthly newsletters, resistance band and pedometer, along with telephone and email contact by program guides. Frequency of telephone contact varied, as some participants requested only phone contact or information via email. Participants generally received between six to 10 phone calls and/or two to five emails over the 6-month period. The protocol of the intervention has been described in detail elsewhere [26]. The control group received baseline and post-intervention questionnaires at the same time as the PANS participants, and both groups were given a small token of appreciation upon completion and return of the postal questionnaires.

The intervention was funded for $\mathrm{A} \$ 400,000$ over a three-year period. The estimated costs to replicate the intervention include salary for a part-time coordinator $(\mathrm{A} \$ 180,000)$ and a research assistant $(\mathrm{A} \$ 150,000)$, intervention materials $(\mathrm{A} \$ 23,500)$ and incentives $(A \$ 7,000)$, postage $(\mathrm{A} \$ 1,500)$, telephone calls $(\mathrm{A} \$ 3,500)$, program guide reimbursement $(\mathrm{A} \$ 6,500)$, guide manuals $(\mathrm{A} \$ 500)$, administration costs $(\mathrm{A} \$ 5,000)$ and printing of questionnaires $(\mathrm{A} \$ 2,500)$. 


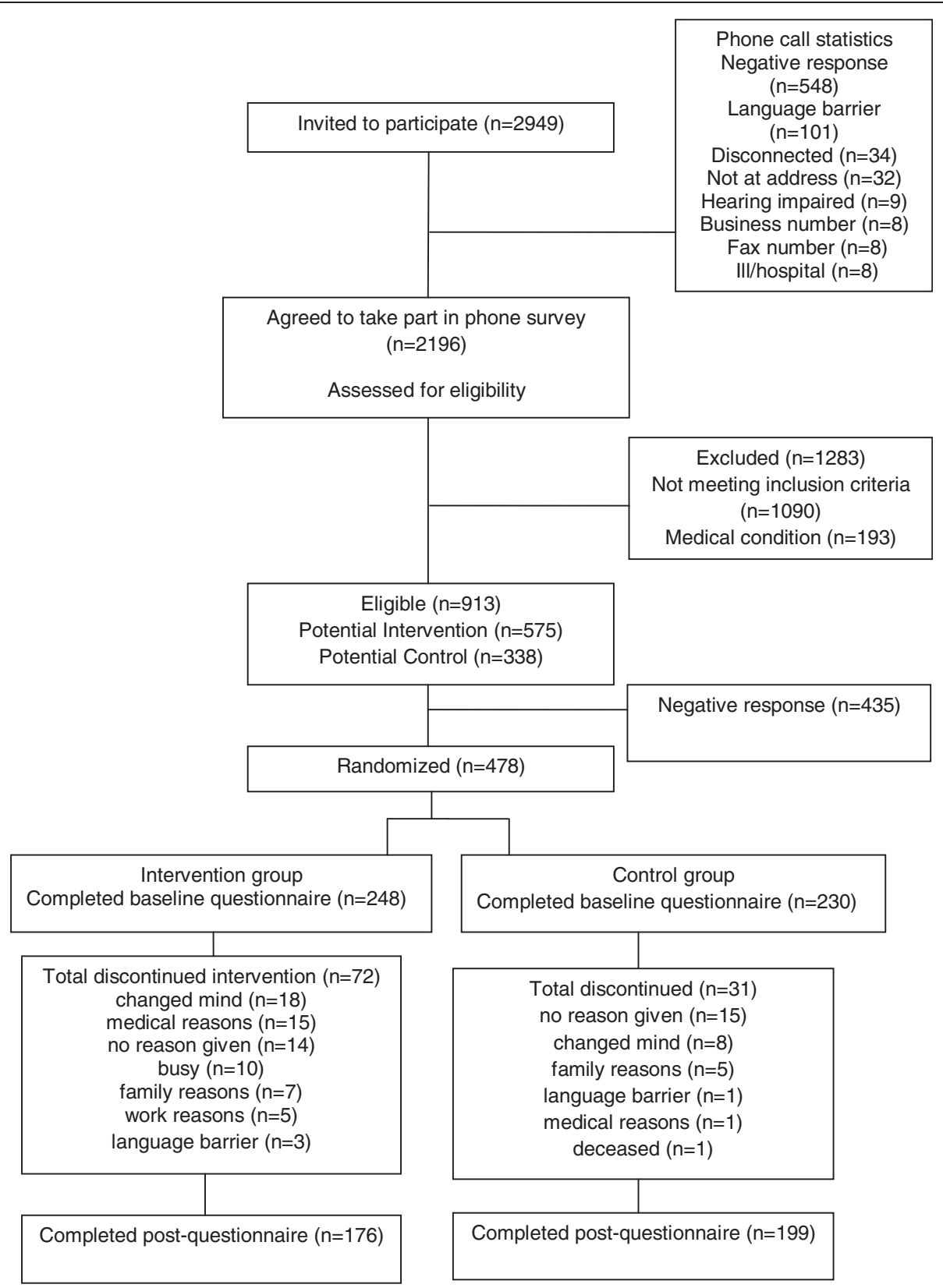

Figure 1 Consort flow chart of intervention participants and controls.

\section{Instrument}

The self-completion questionnaire consisted of previously validated instruments on physical activity and sitting behaviour [35] and nutrition behaviours [36], along with demographic and personal characteristics including gender, age, education level, marital status, tobacco smoking and alcohol consumption. The instrument was reviewed by experts in the field, underwent test re-test, and found to possess moderate to high intra-class correlation (0.62-0.95). The International Physical Activity Questionnaire short-form [35] was used to measure selfreported walking, moderate-intensity physical activity, vigorous-intensity physical activity and sitting time for older adults $[37,38]$. It specifically asked whether a person participated (yes or no) in various types of physical activity and their duration (minimum of 10 minutes). A strength exercise question "During your usual week, on how many days did you do strength activities? How much time did you usually spend doing strength activities on each of these days?" was also appended [39].

Dietary intake behaviours were assessed via a modified version of the Fat and Fibre Barometer [36] to gather specific information on fat intake (e.g. butter, cheese, milk) and fibre-related intake (e.g. cereals, fruit and 
vegetables). Extra questions were added to assess frequency of fruit and vegetable intake, which enabled quantification of the number of days participants consumed at least two servings of fruit or vegetables per week. The content of the intervention emphasised increasing consumption of fruits, vegetables and fibre but reducing the intake of saturated fat.

\section{Statistical analysis}

Descriptive statistics were first applied to summarize the baseline demographic profile and lifestyle characteristics of the sample. Comparisons between intervention and control groups were made across the two time points using independent samples and paired t-tests for continuous outcomes, and chi-square test for categorical outcome variables.

The main outcomes of interest were strength exercise, walking, moderate- and vigorous-intensity physical activity levels, sitting time, fibre intake, fat intake, fat avoidance, frequency of fruit intake and frequency of vegetables intake. In the presence of many zeros (lack of participation by seniors, i.e. $<10$ minutes duration), all physical activity variables were recoded into binary form indicating participation status (yes; no), while sitting time remained as a continuous variable (recorded in minutes per week). For food eating habits, the fibre intake (range 0-28), fat intake (range 0-21) and fat

Table 1 Baseline characteristics of intervention participants and controls

\begin{tabular}{|c|c|c|c|}
\hline Variable & $\begin{array}{l}\text { Intervention } \\
\text { group } \\
(n=176)\end{array}$ & $\begin{array}{l}\text { Control } \\
\text { group } \\
(n=199)\end{array}$ & $p$ value $^{1}$ \\
\hline Age: mean (SD) years & $65.80(2.95)$ & $65.75(3.19)$ & 0.884 \\
\hline Gender: male & $93(52.8 \%)$ & 101 (50.8\%) & 0.686 \\
\hline $\begin{array}{l}\text { Relationship status: with } \\
\text { partner }\end{array}$ & $128(72.7 \%)$ & $159(79.9 \%)$ & 0.102 \\
\hline Work status: working & 77 (43.8\%) & $80(40.2 \%)$ & 0.487 \\
\hline Co-morbidity $^{2}$ : yes & $129(73.3 \%)$ & 139 (69.8\%) & 0.461 \\
\hline $\begin{array}{l}\text { Education level: primary } \\
\text { school }\end{array}$ & $8(4.5 \%)$ & $16(8.0 \%)$ & 0.483 \\
\hline secondary school & $83(47.2 \%)$ & $91(45.7 \%)$ & \\
\hline trade certificate/diploma & $48(27.3 \%)$ & $57(28.6 \%)$ & \\
\hline university & $37(21.0 \%)$ & $35(17.6 \%)$ & \\
\hline Financial struggle: never & $24(13.6 \%)$ & $25(12.6 \%)$ & 0.951 \\
\hline sometimes & $115(65.3 \%)$ & $131(65.8 \%)$ & \\
\hline always & 37 (21.0\%) & $43(21.6 \%)$ & \\
\hline Alcohol drinking: yes & $116(65.9 \%)$ & $137(68.8 \%)$ & 0.545 \\
\hline Smoking status: never & $97(55.1 \%)$ & $94(47.2 \%)$ & 0.283 \\
\hline former & $69(39.2 \%)$ & $94(47.2 \%)$ & \\
\hline current & $10(5.7 \%)$ & $11(5.5 \%)$ & \\
\hline
\end{tabular}

${ }^{1}$ chi-square or $t$ test between intervention and control groups.

${ }^{2}$ presence of at least one of nine common health conditions. avoidance (range 6-30) composite scores were computed based on the corresponding consumption behavioural questions from the Fat and Fibre Barometer, whereas consumption of at least two servings of fruit per week was considered as either infrequent ( 0 to 2 days) or frequent (3 to 7 days), and analogously for vegetables consumption.

To accommodate the inherent correlation of observations taken from the same individual, generalized estimating equation (GEE) models with exchangeable correlation structure were fitted to assess the repeated measures over time, while accounting for the effects of potential confounding factors. All binary outcomes were modelled using logistic GEE. Normal GEE with identity link was applied to fibre intake and fat intake scores, whereas gamma GEE with log link was considered appropriate for modelling the highly skewed sitting time variable and fat avoidance score. All statistical analyses were undertaken in the SPSS package, version 18.

\section{Results}

Characteristics of the sample are summarized in Table 1 which shows the intervention and control groups were similar in terms of demographics and lifestyle at baseline. Overall, the mean age was 66 years, about half were male, the majority of them had a partner and experienced common health conditions. Less than half the seniors completed secondary school but over $40 \%$ were still in the workforce. No differences in reported alcohol drinking and smoking status were found between the intervention and control participants. No adverse events were reported in relation to the intervention.

Process evaluation based on a brief questionnaire indicated good adherence to the program. Participants reported that the booklet encouraged them to think about physical activity (78\%) and nutrition (70\%), with the majority using the exercise chart (74\%) to practise the recommended exercises (62\%). Moreover, the calendar reminded them to consider physical activity (66\%) and nutrition (55\%). About $90 \%$ of the intervention participants reported using the pedometer while $63 \%$ utilised the resistance band to perform strength exercises.

Table 2 compares the physical activity outcomes between the intervention and control groups across the two time points. Both groups were similar in terms of physical activity participation at baseline, except in sitting time. However, significant improvements in these outcomes from baseline to post-program were evident among the intervention participants but not the controls. In particular, the intervention group exhibited significantly higher prevalence of participation in strength exercise and walking than the control group at six months. 
Comparison of nutritional behaviours are summarised in Table 3. All nutritional outcomes were similar between the two groups at baseline. At six months, the intervention participants demonstrated significant increases in fibre intake and fat avoidance, with significantly higher mean scores than the controls. Improvements in prevalence of frequent fruit intake and prevalence of frequent vegetable intake were also observed in the intervention group, though the latter increase appeared marginal. As expected, there was little change in dietary habits among the controls over the six-month period.

Results of the GEE analyses are given in Table 4. After controlling for demographic and other confounding factors, the regression results confirmed significant increases in engagement in strength exercise $(\mathrm{p}<0.001)$, walking $(\mathrm{p}=$ $0.029)$ and vigorous-intensity physical activity $(\mathrm{p}=0.015)$ but not moderate-intensity physical activity $(\mathrm{p}=0.144)$ for the intervention participants relative to the controls. The PANS intervention was also successful in significantly reducing the sitting time of participants through the groupxtime interaction term $(\mathrm{p}<0.001)$. Moreover, positive behavioural changes towards reducing dietary fats were evident in the intervention group in terms of fat avoidance $(\mathrm{p}<0.001)$ and fat intake $(\mathrm{p}=0.021)$ when compared with the controls. The likelihood of frequent fruit intake significantly increased among the PANS participants post-intervention $(\mathrm{p}=0.008)$, but fibre intake and prevalence of frequent vegetable intake did not change significantly after the sixmonth period. The estimated correlations between the repeated observations were substantial which justified the fitting of GEE models.

\section{Discussion}

Appropriate home-based interventions can improve physical activity and nutrition behaviours in insufficiently active
$60-70$ year olds $[33,34,40]$, and are especially useful when they allow for flexibility, with self-tailoring to suit individual pace and needs $[15,33,38]$. The PANS intervention was developed based on a large pilot study [33,34] and offered a practical community-based program for older people. The relatively low cost trial was designed to evaluate the effect of combining physical activity and nutrition on behavioural changes of seniors with low to middle socioeconomic status. The moderate sample sizes provided sufficient statistical power for evaluation of the repeated measures [26]. The overall response rate of $78.5 \%$ was comparable with other randomized controlled trials on seniors $[24,41]$. The main reasons of attrition such as work and family commitments, illness and injuries, were consistent with other studies in the literature [15,24]. The International Physical Activity Questionnaire short-form appears to be useful to assess physical activity behavioural change for older adults. However, objective assessment of physical activity should be considered in future research.

The results from this 6-month home-based intervention for seniors indicated improvements in physical activity and nutritional behaviours among program participants in comparison to the controls. The intervention was shown to be effective and consistent with previous studies in terms of levels of change in physical activity and nutrition behaviours [15], specifically, increases in walking [34,38], participation in strength exercises [15], increases in vigorousintensity physical activity [42], improvements in fruit intake $[15,43]$ and a reduced consumption of fat [15]. However, fibre intake behaviour and the frequency of vegetable intake showed no significant change. The seniors may already maintain a varied and healthy diet with a low consumption of take-away foods at baseline. This could have imposed limitations on further dietary gains, producing a so called "ceiling effect" [34,44].

Table 2 Comparison of physical activity outcomes between intervention participants and controls

\begin{tabular}{|c|c|c|c|c|c|}
\hline \multirow[t]{2}{*}{ Outcome } & \multicolumn{2}{|c|}{ Intervention group $(n=176)$} & \multicolumn{2}{|c|}{ Control group $(n=199)$} & \multirow[t]{2}{*}{ chi-square or $t$ test } \\
\hline & Baseline & Post & Baseline & Post & \\
\hline \multirow[t]{2}{*}{ Strength exercise ${ }^{1}$} & $34(19.3 \%)$ & $70(39.8 \%)$ & $55(27.6 \%)$ & $55(27.6 \%)$ & $p_{2}=0.060 p_{3}=0.013$ \\
\hline & $p_{1}<0.001$ & & $p_{1}=1$ & & \\
\hline \multirow[t]{2}{*}{ Walking ${ }^{1}$} & $152(86.4 \%)$ & $166(94.3 \%)$ & $171(85.9 \%)$ & $173(86.9 \%)$ & $p_{2}=0.903 p_{3}=0.015$ \\
\hline & $p_{1}=0.012$ & & $p_{1}=0.770$ & & \\
\hline \multirow[t]{2}{*}{ Moderate activity ${ }^{1}$} & $124(70.5 \%)$ & $145(82.4 \%)$ & $143(71.9 \%)$ & $154(77.4 \%)$ & $p_{2}=0.764 p_{3}=0.229$ \\
\hline & $p_{1}=0.008$ & & $p_{1}=0.205$ & & \\
\hline \multirow[t]{2}{*}{ Vigorous activity $^{1}$} & $33(18.8 \%)$ & $49(27.8 \%)$ & $55(27.6 \%)$ & $51(25.6 \%)$ & $p_{2}=0.050 p_{3}=0.629$ \\
\hline & $p_{1}=0.044$ & & $p_{1}=0.650$ & & \\
\hline \multirow[t]{2}{*}{ Sitting time: mean (SD) min per week } & $2063(1050)$ & $1708(952)$ & $1691(925)$ & $1734(986)$ & $p_{2}<0.001 p_{3}=0.794$ \\
\hline & $p_{1}<0.001$ & & $p_{1}=0.441$ & & \\
\hline
\end{tabular}

${ }^{1}$ participation of at least 10 minutes.

$p_{1}$ : baseline versus post $\mathrm{p}$ value.

$p_{2}$ : baseline intervention versus baseline control $p$ value.

$p_{3}$ : post intervention versus post control $p$ value. 
Table 3 Comparison of nutritional outcomes between intervention participants and controls

\begin{tabular}{|c|c|c|c|c|c|}
\hline \multirow[t]{2}{*}{ Outcome } & \multicolumn{2}{|c|}{ Intervention group $(n=176)$} & \multicolumn{2}{|c|}{ Control group $(n=199)$} & \multirow[t]{2}{*}{ chi-square or $t$ test } \\
\hline & Baseline & Post & Baseline & Post & \\
\hline \multirow[t]{2}{*}{ Frequent fruit intake $^{1}$} & $153(86.9 \%)$ & $164(93.2 \%)$ & $167(83.9 \%)$ & $163(81.9 \%)$ & $p_{2}=0.250 p_{3}=0.001$ \\
\hline & $p_{1}=0.037$ & & $p_{1}=0.345$ & & \\
\hline \multirow[t]{2}{*}{ Frequent vegetable intake ${ }^{1}$} & $155(88.1 \%)$ & $165(93.8 \%)$ & $170(85.4 \%)$ & $177(88.9 \%)$ & $p_{2}=0.275 p_{3}=0.072$ \\
\hline & $p_{1}=0.047$ & & $p_{1}=0.184$ & & \\
\hline \multirow[t]{2}{*}{ Fibre intake score: range $0-28$, mean (SD) } & $16.77(5.60)$ & $18.07(5.30)$ & $16.14(6.05)$ & $16.74(6.05)$ & $p_{2}=0.300 p_{3}=0.025$ \\
\hline & $p_{1}<0.001$ & & $p_{1}=0.035$ & & \\
\hline \multirow[t]{2}{*}{ Fat avoidance score: range 6-30, mean (SD) } & $21.53(4.83)$ & $22.81(4.34)$ & $21.36(4.78)$ & $21.49(4.77)$ & $p_{2}=0.757 p_{3}=0.009$ \\
\hline & $p_{1}<0.001$ & & $p_{1}=0.953$ & & \\
\hline \multirow[t]{2}{*}{ Fat intake score: range $0-21$, mean (SD) } & $1.84(1.99)$ & $1.63(1.60)$ & $1.47(1.56)$ & $1.60(1.86)$ & $p_{2}=0.280 p_{3}=0.350$ \\
\hline & $p_{1}=0.049$ & & $p_{1}=0.230$ & & \\
\hline
\end{tabular}

${ }^{1}$ consumption of at least two servings on 3 to 7 days per week.

$p_{1}$ : baseline versus post $\mathrm{p}$ value.

$p_{2}$ : baseline intervention versus baseline control $p$ value.

$p_{3}$ : post intervention versus post control $p$ value.

The health benefits of physical activity and its role in preventing many chronic diseases are well established $[6,10,45]$. On the other hand, recent research has suggested that sitting for long periods of time can have a detrimental effect on the body's physiology, with excessive sitting being recognised as a serious health hazard [5]. The PANS intervention was effective in reducing the sitting time of seniors. There is clearly a need for incorporating sitting time within physical activity guidelines [3,46,47], and positive change in sedentary behaviour should be a key component of future intervention programs.

\section{Limitations}

In this study, the data collected from the postal questionnaires were based on self-report, although similar inaccuracies would be expected between the intervention and control groups. Large scale community trials have used self-reported data as valid proxies to reduce cost and attrition rates, and such data have been considered sufficiently reliable for monitoring changes over time [15,48-51] which formed the basis of our evaluation. Self-selection bias was minimized through randomisation, but participation in the home-based intervention was entirely voluntary. Therefore, reporting bias might still be a problem. Furthermore, residual confounding could not be ruled out even though demographic and other factors were controlled for in the GEE regression analyses.

\section{Conclusions}

The PANS participants improved their physical activity and dietary habits in comparison to the controls, confirming that a low-cost, home-based physical activity and nutrition

Table 4 Regression analysis of outcomes before and after intervention $(n=375)$

\begin{tabular}{lllll}
\hline Outcome $^{-}$ & Coefficient $^{{ }^{1}}$ & $\mathbf{9 5 \%}$ Confidence interval & $\boldsymbol{p ~ v a l u e ~}^{\text {Correlation }^{2}}$ \\
Strength exercise $^{3}$ & 1.075 & $(0.559,1.591)$ & $<0.001$ & 0.417 \\
Walking $^{3}$ & 0.909 & $(0.094,1.724)$ & 0.029 & 0.314 \\
Moderate activity $^{3}$ & 0.416 & $(-0.142,0.974)$ & 0.144 & 0.387 \\
Vigorous activity $^{3}$ & 0.664 & $(0.128,1.199)$ & 0.015 & 0.405 \\
Sitting time $^{4}$ & -0.215 & $(-0.312,-0.117)$ & $<0.001$ & 0.583 \\
Frequent fruit intake $^{3}$ & 0.921 & $(0.236,1.607)$ & 0.008 & 0.400 \\
Frequent vegetable intake $^{3}$ & 0.424 & $(-0.403,1.251)$ & 0.314 & 0.275 \\
Fibre intake $^{5}$ & 0.716 & $(-0.115,1.546)$ & 0.091 & 0.742 \\
Fat avoidance $^{4}$ & 0.057 & $(0.028,0.085)$ & $<0.001$ & 0.843 \\
Fat intake $^{5}$ & -0.345 & $(-0.639,-0.051)$ & 0.021 & 0.637 \\
\hline
\end{tabular}

1 effect of group by time interaction, adjusted for age, gender, relationship status, work status, co-morbidity, education level, financial struggle, alcohol drinking, smoking status, group (intervention/control) and time (baseline/post).

2 exchangeable correlation estimate.

${ }^{3}$ logistic generalized estimating equation model.

${ }^{4}$ gamma generalized estimating equation model with log link.

5 normal generalized estimating equation model with identity link. 
program tailored for insufficiently active, low to middle income seniors can produce effective behavioural changes. A follow-up study is recommended to confirm the adherence of the positive behavioural changes beyond six months. It would also be useful to replicate the program both in the community and in other settings where seniors reside such as retirement villages.

\section{Abbreviations}

GEE: generalized estimating equation; PANS: physical activity and nutrition for seniors.

\section{Competing interests}

The authors declare that they have no competing interests.

\section{Authors' contributions}

$L B$ conducted the trial and drafted the manuscript. PAH coordinated the study. PAH, AHL, JJ, DAK, APH and ASA contributed to conception and study design and revised the manuscript. LX and AHL performed statistical analysis and interpreted the data. All authors read and approved the final manuscript.

\section{Acknowledgements}

This study was funded by a three-year Australian National Health and Medical Research Council grant (project number 533501). The authors are grateful to Maria Pasalich and Choon Cheong Leong for technical assistance. Thanks are also due to the seniors who participated in the study.

\section{Author details}

${ }^{1}$ School of Public Health, Curtin University, Perth, WA, Australia. ${ }^{2}$ School of Physical and Mathematical Sciences, Nanyang Technological University, Nanyang, Singapore. ${ }^{3}$ Centre for Behavioural Research in Cancer Control, Curtin University, Perth, WA, Australia. ${ }^{4}$ Mater Mother's Hospital/Mater Medical Research Institute (MMRI), Griffith University/Griffith Health Institute (GHI), Griffith, QLD, Australia. ${ }^{5}$ Centre for Public Health Nutrition Research, Division of Clinical Population Sciences and Education, University of Dundee, Scotland, UK.

Received: 5 March 2012 Accepted: 24 January 2013

Published: 31 January 2013

\section{References}

1. Thogersen-Ntoumani C, Loughren E, Duda J, Fox K, Kinnafick F-E: "Step by Step": A feasibility study of a lunchtime walking intervention designed to increase walking, improve mental well-being and work performance in sedentary employees: Rationale and study design. BMC Public Health 2010, 10(1):578.

2. Australian Bureau of Statistics: National Health Survey. Canberra: Summary of Results; 2009

3. U.S: Department of Health and Human Services. Washingtion, DC: Physical Activity Guidelines for Americans; 2008.

4. Centers for Disease Control and Prevention: U.S. Physical Activity Statistics. USA Government: State Demographic Data Comparison Atlanta, USA. Department of Health and Human Services; 2007.

5. Hamilton M, Healy G, Dunstan D, Zderic T, Owen N: Too little exercise and too much sitting: Inactivity physiology and the need for new recommendations on sedentary behavior. Current Cardiovascular Risk Reports 2008, 2(4):292-298.

6. Hamilton MT, Hamilton DG, Zderic TW: Role of Low Energy Expenditure and Sitting in Obesity, Metabolic Syndrome, Type 2 Diabetes, and Cardiovascular Disease. Diabetes 2007, 56(11):2655-2667.

7. Hu FB: Television watching and other sedentary behaviors in relation to risk of obesity and type 2 diabetes mellitus in women. JAMA (Chicago, III) 2003, 289(14):1785

8. Katzmarzyk PT: Sitting time and mortality from all causes, cardiovascular disease, and cancer. Medicine and science in sports and exercise 2009, 41(5):998.

9. Patel AV: Leisure time spent sitting in relation to total mortality in a prospective cohort of US adults. American journal of epidemiology 2010, 172(4):419.
10. Balboa-Castillo T, León-Muñoz LM, Graciani A, Rodríguez-Artalejo F, GuallarCastillón P: Longitudinal association of physical activity and sedentary behavior during leisure time with health-related quality of life in community-dwelling older adults. 27 June 2011 edition 2011.

11. Touvier M, Bertrais S, Charreire H, Vergnaud AC, Hercberg S, Oppert JM: Changes in leisure-time physical activity and sedentary behaviour at retirement: a prospective study in middle-aged French subjects. 2010.

12. National Health and Medical Research Council: Canberra, ACT: Australian Dietary Guidelines - draft; 2011.

13. World Health Organization: Geneva, Switzerland: World Health Statistics; 2012.

14. Kearney J: Food consumption trends and drivers. Phylosophical Transactions of the Royal Society Biological Sciences 2010, 365:2793-2807.

15. Morey MC, Snyder DC, Sloane R, Cohen HJ, Peterson B, Hartman TJ, Miller P, Mitchell DC, Demark-Wahnefried W: Effects of home-based diet and exercise on functional outcomes among older, overweight long-term cancer survivors. The Journal of the American Medical Association 2009, 301(18):1883-1891.

16. Tudor-Locke C, Bell RC, Myers AM, Harris SB, Ecclestone NA, Lauzon N, Rodger NW: Controlled outcome evaluation of the First Step Program: a daily physical activity intervention for individuals with type II diabetes. Int J Obes Relat Metab Disord 2003, 28(1):113-119.

17. Clark PG, Rossi JS, Greaney ML, Riebe DA, Greene GW, Saunders SD, Lees FD, Nigg CR: Intervening on Exercise and Nutrition in Older Adults: The Rhode Island SENIOR Project. Journal of Aging and Health 2005, 17(6):753-778.

18. Bernstein MA, Nelson ME, Tucker KL, Layne J, Johnson E, Nuernberger A, Castaneda C, Judge JO, Buchner D, Singh MF: A home-based nutrition intervention to increase consumption of fruits, vegetables, and calciumrich foods in community dwelling elders. Journal of the American Dietetic Association 2002, 102(10):1421-1422.

19. Villareal DT, Chode S, Parimi N, Sinacore DR, Hilton T, Armamento-Villareal R, Napoli N, Qualls C, Shah K: Weight Loss, Exercise, or Both and Physical Function in Obese Older Adults. New England Journal of Medicine 2011, 364(13):1218-1229.

20. McClure J, Catz S, Ludman E, Richards J, Riggs K, Grothaus L: Feasibility and acceptability of a multiple risk factor intervention: The Step Up randomized pilot trial. BMC Public Health 2011, 11(1):167.

21. Prochaska JJ, Nigg CR, Spring B, Velicer WF, Prochaska JO: The benefits and challenges of multiple health behavior change in research and in practice. Preventive medicine 2008, 50(1-2):26-29.

22. Cavill JL, Jancey J, Howat P: Review and recommendations for online physical activity and healthy weight programs targeted at over $40 \mathrm{~s}$. Global Health Promotion, in press.

23. Bourke L, Thompson G, Gibson DJ, Daley A, Crank H, Adam I, Shorthouse A, Saxton J: Pragmatic Lifestyle Intervention in Patients Recovering From Colon Cancer: A Randomized Controlled Pilot Study. Archives of Physical Medicine and Rehabilitation 2011, 92(5):749-755.

24. Cox KL, Burke V, Beilin LJ, Derbyshire AJ, Grove JR, Blanksby BA, Puddey IB: Short and long-term adherence to swimming and walking programs in older women-the Sedentary Women Exercise Adherence Trial (SWEAT 2). Preventive Medicine 2008, 46(6):511-517.

25. Matthews F, Marshall P, Stone D: Achieving Health and Human Development. Macmillan Education Australia: Units 1 and 2; 2009.

26. Burke L, Jancey J, Howat P, Lee A, Kerr D, Shilton T, Hills A, Anderson A: Physical activity and nutrition program for seniors (PANS): protocol of a randomized controlled trial. BMC Public Health 2010, 10(1):751.

27. Australian Bureau of Statistics: Socio-Economic Indexes for Areas (SEIFA), Data only, 2006. Canberra: Australian Bureau of Statistics; 2008.

28. Brown WJ, Moorhead GE, Marshall AL: Choose health:Be Active:A physical activity guide for older Australians. 3rd edition. Canberra: Commonwealth of Australia and repatriation Commission; 2008

29. Artinian NT, Fletcher GF, Mozaffarian D, Kris-Etherton P, Van Horn L, Lichtenstein AH, Kumanyika S, Kraus WE, Fleg JL, Redeker NS, et al: Interventions to Promote Physical Activity and Dietary Lifestyle Changes for Cardiovascular Risk Factor Reduction in Adults. A scientific statement from the American Heart Association. Circulation 2010, 122(4):406-441.

30. Bandura A: Social foundations of thought and action. 3rd edition. Englewood Cliffs, NJ: Prentice Hall; 1986.

31. Green LW, Kreuter MW: Health program planning: an educational and ecological approach. 4th edition. New York: McGraw Hill; 2005.

32. Rosenstock IM, Strecher VJ, Becker MH: Social Learning Theory and the Health Belief Model. Health Education \& Behavior 1988, 15(2):175-183. 
33. Burke L, Howat P, Lee A, Jancey J, Kerr D, Shilton T: Development of a nutrition and physical activity booklet to engage seniors. BMC Research Notes 2008, 1(77):1-7.

34. Lee A, Jancey J, Howat P, Burke L, Kerr D, Shilton T: Effectiveness of a home-based physical activity and nutrition pilot program for seniors. Journal of Obesity 2011, 2011:8.

35. Craig C, Marshall M, Sjostrom A, Bauman A, Booth B, Ainsworth M, Pratt U, Ekelund A, Yngve J, Sallis J, Oja P: International physical activity questionnaire: 12 country reliability and validity. Medical Science Sports Exercise 2003, 35(8):1381-1395.

36. Wright JL, Scott JA: The Fat and Fibre Barometer, a short food behaviour questionnaire: reliability, relative validity and utility. Australian Journal of Nutrition and Dietetics 2000, 57(1):33-39.

37. Jancey J, Lee A, Howat P, Clarke A, Wang K, Shilton T: Reducing attrition in physical activity programs for older adults. Journal of Aging and Health 2007, 15(2):152-165.

38. Jancey J, Clarke A, Howat P, Lee A, Shilton T, Fisher J: A physical activity program to mobilize older people: A practical and sustainable approach. The Gerontologist 2008, 48(2):251-257.

39. Haskell W, Lee I, Pate R, Powell K, Blair S, Franklin B, Macera C, Heath G, Thompson P, Bauman A: Physical activity and public health: updated recommendations for adults from the American College of Sports Medicine and American Heart Association. Circulation 2007, 116:1-13.

40. Goodpaster BH, DeLany JP, Otto AD, Kuller L, Vockley J, South-Paul JE, Thomas SB, Brown J, McTigue K, Hames KC, Lang W, Jakicic JM: Effects of Diet and Physical Activity Interventions on Weight Loss and Cardiometabolic Risk Factors in Severely Obese Adults. JAMA: The Journal of the American Medical Association 2010, 304(16):1795-1802.

41. Greaney ML, Riebe D, Ewing Garber C, Rossi JS, Lees FD, Burbank PA, Nigg $\mathrm{CR}$, Ferrone $\mathrm{CL}$, Clark PG: Long-term effects of a stage-based intervention for changing exercise intentions and behavior in older adults. The Gerontologist 2008, 48(3):358.

42. Wilcox S, Dowda M, Leviton LC, Bartlett-Prescott J, Bazzarre T, CampbellVoytal K, Carpenter RA, Castro CM, Dowdy D, Dunn AL, Griffin SF, Guerra M, King AC, Ory MG, Rheaume C, Tobnick J, Wegley S: Active for Life: Final Results from the Translation of Two Physical Activity Programs. American Journal of Preventive Medicine 2008, 35(4):340-351.

43. Greene GW, Fey-Yensan N, Padula C, Rossi SR, Rossi JS, Clark PG: Change in fruit and vegetable intake over 24 months in older adults: results of the SENIOR project intervention. The Gerontologist 2008, 48(3):378.

44. de Vreede PL, van Meeteren NL, Samson MM, Wittink HM, Duursma SA, Verhaar HJ: The Effect of Functional Tasks Exercise and Resistance Exercise on Health-Related Quality of Life and Physical Activity. Gerontology 2007, 53(1):9

45. Bassuk SS, Manson JE: Epidemiological evidence for the role of physical activity in reducing risk of type 2 diabetes and cardiovascular disease. Journal of Applied Physiology 2005, 99(3):1193-1204.

46. Warburton D, Charlesworth S, Ivey A, Nettlefold L, Bredin S: A systematic review of the evidence for Canada's Physical Activity Guidelines for Adults. International Journal of Behavioral Nutrition and Physical Activity 2010, 7(1):39.

47. World Health Organisation: Global Recommendations on Physical Activity for Health. Switzerland; 2012

48. Burton NW, Brown W, Dobson A: Accuracy of body mass index estimated from self-reported height and weight in mid-aged Australian women. Australian and New Zealand Journal of Public Health 2010, 34(6):4.

49. Dhaliwal S, Howat P, Bejoy T, Welborn T: Self-reported weight and height for evaluating community obesity studies. American Journal of Health Behavior 2010, 34(4):489-499.

50. Eakin E, Reeves M, Lawler S, Graves N, Oldenburg B, Del Mar C, Wilke K, Winkler E, Barnett A: Telephone Counseling for Physical Activity and Diet in Primary Care Patients. American Journal of Preventive Medicine 2009, 36(2):142-149.

51. Jancey JM, Clarke A, Howat PA, Maycock B, Lee AH: Perceptions of physical activity by older adults: A qualitative study. Health Education Journal 2009, 68(3):196-206.

doi:10.1186/1479-5868-10-14

Cite this article as: Burke et al:: Physical activity and nutrition behavioural outcomes of a home-based intervention program for seniors: a randomized controlled trial. International Journal of Behavioral Nutrition and Physical Activity 2013 10:14

\section{Submit your next manuscript to BioMed Central and take full advantage of:}

- Convenient online submission

- Thorough peer review

- No space constraints or color figure charges

- Immediate publication on acceptance

- Inclusion in PubMed, CAS, Scopus and Google Scholar

- Research which is freely available for redistribution 